\title{
Diagnostic Value of Procalcitonin in Predicting Bacteremia in Intensive Care Unit
}

\author{
Tuna Demirdal, Pinar Sen, Salih Atakan Nemli \\ Department of Infectious Diseases and Clinical Microbiology, Izmir Katip Celebi University, Ataturk Training and Research Hospital, Izmir, Turkey
}

\section{Abstract}

Background and Aims: Several biomarkers are used in the diagnosis of bacteremia. Procalcitonin (PCT) is more specific than other biomarkers in differentiating bacterial and nonbacterial inflammation. It was aimed to evaluate the diagnostic and prognostic value of PCT in bacteremic patients in Intensive Care Unit (ICU). Materials and Methods: A total of 156 patients diagnosed with noninfectious systemic inflammatory response syndrome, sepsis, and severe sepsis/septic shock in ICU between December 2014 and July 2015 were evaluated in this prospective cohort study. Results: The study group consisted of 64 (41\%) bacteremic patients and the control group consisted of 92 (59\%) nonbacteremic patients. The overall mortality rate was $60.3 \%$. Although PCT levels in the bacteremic group $(11.9 \pm 21.5 \mathrm{ng} / \mathrm{dL})$ were higher than nonbacteremic group $(5.9 \pm 11.5 \mathrm{ng} / \mathrm{dL})$, this difference was not significant $(P=0.168)$. The mean levels of PCT in bacteremic patients with Gram-negative bacteria were $16.3 \pm 27.6 \mathrm{ng} / \mathrm{dL}$, whereas Gram-positive bacteria were $7.3 \pm 10.7 \mathrm{ng} / \mathrm{dL}(P=0.145)$. The mean PCT levels were significantly higher in nonsurvivors compared to survivors $(10.1 \pm 18.0$ vs. $5.7 \pm 13.7 \mathrm{ng} / \mathrm{dL} ; P<0.001)$. Conclusions: PCT may be an effective biomarker for diagnosing sepsis and predicting disease severity and mortality. There is a need for further well-designed studies to confirm the diagnostic and prognostic value of PCT in septic patients in critical care.

Keywords: Bacteremia, Internal Care Unit, procalcitonin, sepsis

\section{INTRODUCTION}

Sepsis is dysregulated and uncontrolled inflammatory response against infection. The incidence of severe sepsis and septic shock has been increasing rapidly in recent years, especially in Intensive Care Units (ICUs). ${ }^{[1]}$ Sepsis and septic shock are also the most common causes of death in ICU. ${ }^{[2]}$ Early diagnosis of sepsis and rapid initiation of appropriate therapy are the most important factors affecting the clinical course and reducing mortality rate. Therefore, accurate recognition and appropriate antibiotic treatment are lifesaving in critically ill patients. Determination of the causative agent is important for the selection of appropriate antibiotic therapy. ${ }^{[3]}$ The isolation of microorganisms is considered to be the gold standard for the diagnosis of sepsis. ${ }^{[4]}$ However, blood culture positivity can be detected approximately $30 \%$ of bacteremic patients and positive blood cultures may not be identified until 48-72 h. ${ }^{[4,5]}$ The sensitivity and specificity of routine laboratory tests are inadequate to discriminate between infectious and noninfectious conditions.

\begin{tabular}{|l|l|}
\hline \multicolumn{3}{|c|}{ Access this article online } \\
\hline Quick Response Code: & Website: \\
& www.ijccm.org \\
\cline { 2 - 2 } & \\
&
\end{tabular}

Procalcitonin (PCT) is a prehormone of calcitonin, and it is secreted from $\mathrm{C}$ cells of the thyroid gland. ${ }^{[6]}$ This hormone is released from thyroid $\mathrm{C}$ cells, lung, liver, intestines, and pancreatic neuroendocrine cells during inflammation. ${ }^{[7]} \mathrm{PCT}$ is converted to calcitonin completely; however, the conversion is inhibited by the effect of released cytokines in inflammation. ${ }^{[6]}$ Therefore, this molecule has negligible serum concentration in healthy individuals, and it has been found that PCT was elevated, especially in bacterial inflammation, sepsis, and organ failure. ${ }^{[8]}$ Hence, PCT is more specific than other biomarkers in differentiating bacterial and nonbacterial inflammation. ${ }^{[9]}$ The aim of this study was to evaluate the value of PCT in predicting bacteremia in ICU.

Address for correspondence: Prof. Pinar Sen, Izmir Katip Celebi University, Ataturk Research and Training Hospital, 35360, Karabaglar, Izmir, Turkey. E-mail: pinarozdemirsen@gmail.com

This is an open access article distributed under the terms of the Creative Commons Attribution-NonCommercial-ShareAlike 3.0 License, which allows others to remix, tweak, and build upon the work non-commercially, as long as the author is credited and the new creations are licensed under the identical terms.

For reprints contact: reprints@medknow.com

How to cite this article: Demirdal T, Sen P, Nemli SA. Diagnostic value of procalcitonin in predicting bacteremia in Intensive Care Unit. Indian J Crit Care Med 2018;22:78-84. 


\section{Materials and Methods}

\section{Study design}

The study protocol was approved by local ethics committee (Date: 10.09.2014, Decision no: 140). Informed written consent was obtained from first-degree relatives of patients.

We conducted a single-center, prospective cohort study at the 1055-bed tertiary referral care center containing an 86-bed adult ICU. A total of 225 consecutive patients diagnosed with noninfectious systemic inflammatory response syndrome (SIRS), sepsis, and severe sepsis/septic shock in the anesthesia ICU were evaluated between December 2014 and July 2015. European Society of Intensive Care Medicine (ESICM) consensus criteria (2012) were used in the diagnosis of sepsis. The disease severity was evaluated with the Acute Physiology and Chronic Health Evaluation (APACHE) II Score and the Sequential Organ Failure Assessments (SOFA) Score. Comorbidities were assessed using the Charlson Comorbidity Index.

The exclusion criteria were age $<18$ years, contaminated blood cultures, fungemia, human immunodeficiency virus infection and/or acquired immunodeficiency syndrome, hematological malignancies, chemotherapy, pregnancy, and cardiogenic shock.

Blood cultures and blood samples for PCT and other infection markers (C-reactive protein [CRP] and sedimentation) were taken within the first $24 \mathrm{~h}$ of ICU admission. A total of 156 patients who met inclusion criteria were enrolled in the study. The data of the patients including demographic characteristics, vital signs, comorbidity, cause of hospitalization, length of stay in the ICU, prior antibiotic use, mechanical ventilation, source of infection, mortality rates, The APACHE II scores, SOFA scores, and Charlson comorbidity index scores were recorded. Laboratory parameters, vital signs, and scoring systems were based on maximum deviation from normal values and physiologic variables within $24 \mathrm{~h}$ of admission to the ICU.

\section{Laboratory examinations}

Blood cultures were drawn from the patients within the first $24 \mathrm{~h}$ of their admission to the ICU before the first dose of empiric antibiotics were administered, if needed. Positive blood cultures were identified using the BACTEC FX automatic blood culture detection system (Becton Dickinson, Sparks, MD, USA) in the microbiology laboratory. The antimicrobial susceptibility of isolated strains was determined using the Phoenix Automated Microbiology System (BD Diagnostic Systems, Sparks, MD, USA) in accordance with European Committee on Antimicrobial Susceptibility Testing (EUCAST) criteria. ${ }^{[10]}$ Bacteremia was defined as the isolation of any bacterial species in one or two blood cultures. Coagulase-negative staphylococci, Bacillus spp., Propionibacterium acnes, Corynebacterium spp., and Micrococcus spp. were considered as contaminants when obtained in a single blood culture. However, determining of this organism in both blood cultures with same sensitivity was considered as true positive.

Venous blood samples $(5 \mathrm{cc})$ were obtained simultaneously with blood cultures to determine the level of PCT and other infection markers on the $1^{\text {st }}$ day after admission. The blood was centrifuged at $3500 \mathrm{rpm}$ for $5 \mathrm{~min}$, and PCT levels were analyzed immediately. The lower detection limit was $0.05 \mathrm{ng} / \mathrm{dL}$.

\section{Statistical analysis}

Statistical analysis was performed to determine the diagnostic and prognostic value of PCT and other infection markers between the groups for predicting bacteremia and mortality using SPSS (version 22) software (IBM SPSS, USA) package with $95 \%$ confidence. Chi-square test, Fisher's exact Chi-square test, trend test, and Mann-Whitney U-test were used for the comparison of categorical data between the groups. The receiver operating characteristic (ROC) curves analyzes, sensitivity and specificity values were calculated using MedCalc version 14 (MedCalc Software) in determining cutoff values and diagnostic and prognostic performance of PCT. $P<0.05$ was considered statistically significant.

\section{RESULTS}

\section{Study population}

A total of 226 patients admitted to the anesthesia ICU were evaluated prospectively during the 2014-2015 study period. Among these patients, 70 (30.9\%) patients were excluded from the study due to fungemia $(n=2,0.8 \%)$, hematological malignancies $(n=1,0.4 \%)$, cardiogenic shock $(n=33,14.6 \%)$, and blood culture contamination $(n=34,15 \%)$ [Figure 1]. A total of 156 patients who had been diagnosed as noninfectious SIRS ( $n=17,10.9 \%$ ), sepsis $(n=69,44.2 \%)$, and severe sepsis/septic shock ( $n=70,44.9 \%)$ were included in the study. The study group consisted of $64(41 \%)$ bacteremic patients. The control group consisted of $92(59 \%)$ nonbacteremic patients. The mean age was $60.5 \pm 16.7$ years. Of these patients, $99(63.5 \%)$ were male. Demographic and clinical findings of the groups are presented in Table 1.

Gender distribution was similar in both groups; however, female patients with a positive blood culture were older $(64.9 \pm 18.7$ years $)$ than male patients with bacteremia $(56.9 \pm 16.4$ years $)(P=0.023)$.

\section{Distribution of microorganisms}

Blood cultures were positive in 64 patients, 31 (48.4\%) of them were Gram-positive and 37 (57.8\%) of them were Gram-negative bacteria, respectively (Escherichia coli $[n=13,20.3 \%]$, coagulase-negative staphylococci $[n=12,18.8 \%]$, Acinetobacter baumannii $[n=11,17.2 \%]$, Staphylococcus aureus $[n=9,14.1 \%]$, Klebsiella spp. [ $n=4,6.3 \%]$, Stenotrophomonas maltophilia $[n=3,4.7 \%]$, Enterococcus faecalis [ $n=3,4.7 \%]$, Enterococcus faecium $[n=2,3.1 \%]$, Gemella spp. [ $n=2,3.1 \%]$, Pseudomonas aeruginosa $[n=1,1.6 \%]$, 


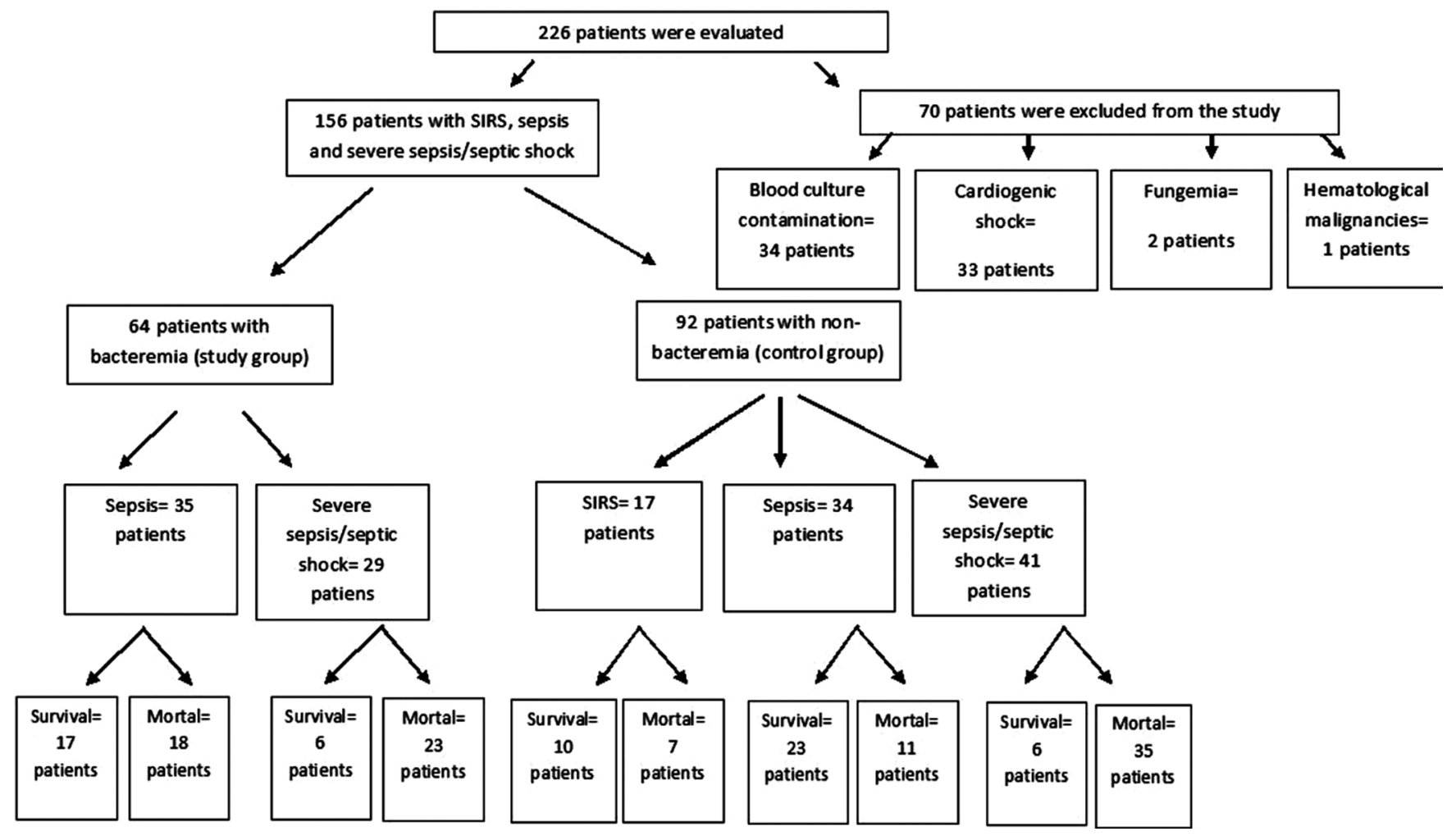

Figure 1: Flowchart of enrolled patients

and others $[n=8,12.8 \%])$. Multiple positive blood cultures were found in $5(7.8 \%)$ patients.

\section{Source of infections}

Source of infections was determined as follows: lower respiratory tract infections $(n=94,60.3 \%)$, intra-abdominal infections $(n=43,27.6 \%)$, urinary tract infections $(n=17,10.9 \%)$, skin and soft-tissue infections $(n=16,10.3 \%)$, catheter infections $(n=5,3.2 \%)$, central nervous system infections $(n=2,1.3 \%)$, and bone and joint infections $(n=1,0.6 \%)$. Multiple foci of infection were detected in $22(14.1 \%)$ patients.

\section{Hospitalizations}

The average ICU length of stay in bacteremic patients was $39.8 \pm 54.7$ days and in the nonbacteremic group was $17.4 \pm 34.6$ days. ICU length of stay was significantly higher in the presence of bacteremia $(P=0.015)$. Length of stay was $36.1 \pm 51.7$ days in patients diagnosed with sepsis and $14.2 \pm 22.3$ days in severe sepsis/septic shock patients. Length of hospitalization was significantly higher in septic patients with bacteremia than nonbacteremic septic patients $(P=0.001)$. In contrast, there was no significant difference in hospital length of stay in bacteremic patients with severe sepsis/septic shock compared to nonbacteremic severe sepsis/septic shock patients $(P=0.924)$.

Diagnostic value of procalcitonin and C-reactive protein The difference in PCT and CRP levels was not significant between the bacteremic and nonbacteremic group $(P=0.168$ and $P=0.343)$ [Table 1]. Although PCT levels in the bacteremic group $(11.9 \pm 21.5 \mathrm{ng} / \mathrm{dL})$ were higher than in the nonbacteremic group $(5.9 \pm 11.5 \mathrm{ng} / \mathrm{dL})$, this difference was not significant $(P=0.168)$ [Table 1]. The Area under the curve (AUC) value for PCT was 0.565 (95\% confidence interval $[\mathrm{CI}]: 0.470-0.660)$ with the cutoff point of 9.3 in predicting bacteremic patients. Applying ROC curve at the cutoff point of 9.3 , PCT yielded $34.9 \%$ sensitivity and $82.6 \%$ specificity [Table 2 and Figure 2].

Mean PCT levels in bacteremic patients with Gram-negative bacteria were $16.3 \pm 27.6 \mathrm{ng} / \mathrm{dL}$, whereas Gram-positive bacteremia mean PCT levels were $7.3 \pm 10.7 \mathrm{ng} / \mathrm{dL}$, and the statistical difference was not significant between the groups $(P=0.145)$. The AUC of PCT was 0.588 (95\% CI: 0.444-0.732) with a cutoff 0.4 in terms of differentiating Gram-positive and Gram-negative bacteremia [Figure 2]. CRP levels did not differ significantly between Gram-positive $(18.8 \pm 8.4 \mathrm{mg} / \mathrm{dl})$ and negative $(18.4 \pm 9.6 \mathrm{mg} / \mathrm{dl})$ bacteremia $(P=0.662)$.

A statistically significant difference was found between SIRS $(0.9 \pm 0.8 \mathrm{ng} / \mathrm{dL})$, sepsis $(4.2 \pm 6.9 \mathrm{ng} / \mathrm{dL})$, and severe sepsis/septic shock $(14.2 \pm 22.4 \mathrm{ng} / \mathrm{dL})$ patients in terms of mean PCT levels $(P<0.001, P=0.001$, and $P<0.001)$. Levels of PCT were found to be significantly higher in septic patients $(P<0.001)$. The cutoff point for PCT was 2 . The calculated AUC for PCT was 0.764 (95\% CI: 0.670-0.857) in predicting septic patients. Applying the ROC curve at the cutoff point of 2, PCT yielded 56.8\% sensitivity and $94.1 \%$ specificity [Table 2 and Figure 2]. 


\begin{tabular}{|c|c|c|c|}
\hline & Bacteremic group $(n=64), n(\%)$ & Nonbacteremic group ( $n=92), n(\%)$ & $P$ \\
\hline Male & $41(26.3)$ & $58(37.2)$ & \\
\hline Female & $23(14.7)$ & $34(21.8)$ & \\
\hline Age (years, mean \pm SD) & $59.8 \pm 17.6$ & $61.0 \pm 16.1$ & 0.837 \\
\hline History of antibiotic use & $26(16.7)$ & $32(20.6)$ & \\
\hline Length of stay (days, mean \pm SD) & $39.8 \pm 54.7$ & $17.5 \pm 34.6$ & 0.015 \\
\hline \multicolumn{4}{|l|}{ Severity of sepsis } \\
\hline SIRS & - & $17(18.5)$ & \\
\hline Sepsis & $35(54.7)$ & $34(37.0)$ & 0.028 \\
\hline Severe sepsis/septic shock & $29(45.3)$ & $41(44.6)$ & 0.926 \\
\hline \multicolumn{4}{|l|}{ Comorbidity } \\
\hline Diabetes mellitus & $19(29.7)$ & $24(26.1)$ & 0.621 \\
\hline Chronic heart failure & $6(9.4)$ & $12(13.0)$ & 0.481 \\
\hline Hypertension & $20(31.3)$ & $33(35.9)$ & 0.549 \\
\hline Chronic renal disease & $10(15.6)$ & $11(12.0)$ & 0.549 \\
\hline Solid organ malignancy & $5(7.8)$ & $8(8.7)$ & 0.844 \\
\hline Chronic lung disease & $5(7.8)$ & $22(23.9)$ & 0.009 \\
\hline Cardiac arrhythmia & $2(3.1)$ & $11(12.0)$ & 0.049 \\
\hline Coronary heart disease & $8(12.5)$ & $17(18.5)$ & 0.317 \\
\hline Cerebrovascular disease & $8(12.5)$ & $8(8.7)$ & 0.441 \\
\hline \multicolumn{4}{|l|}{ Management } \\
\hline Medical patients & $37(57.8)$ & $51(55.4)$ & 0.768 \\
\hline Surgery patients & $27(42.2)$ & $41(44.6)$ & \\
\hline Mechanical ventilation & $48(75.0)$ & $76(82.6)$ & 0.247 \\
\hline Vasopressor use & $29(45.3)$ & $41(44.6)$ & 0.926 \\
\hline \multicolumn{4}{|l|}{ Laboratory findings (mean \pm SD) } \\
\hline White blood cell count $(\mathrm{K} / \mathrm{uL})$ & $13,169.4 \pm 7427.0$ & $15,404.8 \pm 9105.5$ & 0.133 \\
\hline Hemoglobin $(\mathrm{g} / \mathrm{dL})$ & $10.0 \pm 1.7$ & $10.4 \pm 2.1$ & 0.157 \\
\hline Eosinophil (K/uL) & $118.1 \pm 201.9$ & $72.9 \pm 110.6$ & 0.181 \\
\hline Platelet $(\mathrm{K} / \mathrm{uL})$ & $206,578.1 \pm 135,123.6$ & $241,608.7 \pm 143,198.3$ & 0.141 \\
\hline Sedimentation $(\mathrm{mm} / \mathrm{h})$ & $59.9 \pm 31.1$ & $57.1 \pm 33.5$ & 0.551 \\
\hline $\mathrm{CRP}(\mathrm{mg} / \mathrm{dl})$ & $18.6 \pm 8.9$ & $17.5 \pm 10.1$ & 0.343 \\
\hline Procalcitonin (ng/dL) & $11.9 \pm 21.5$ & $5.9 \pm 11.5$ & 0.168 \\
\hline \multicolumn{4}{|l|}{ Mortality } \\
\hline Overall mortality & $41(43.6)$ & $53(56.4)$ & 0.418 \\
\hline 28-day mortality & $29(36.7)$ & $50(63.3)$ & 0.002 \\
\hline APACHE II score $($ mean \pm SD) & $24.5 \pm 8.9$ & $24.5 \pm 8.9$ & 0.891 \\
\hline SOFA score $($ mean $\pm \mathrm{SD})$ & $9.4 \pm 4.1$ & $9.1 \pm 4.3$ & 0.804 \\
\hline Charlson comorbidity index $($ mean $\pm \mathrm{SD})$ & $5.4 \pm 3.5$ & $5.4 \pm 3.6$ & 0.830 \\
\hline
\end{tabular}

SD: Standard deviation; SIRS: Systemic inflammatory response syndrome; CRP: C-reactive protein; APACHE: Acute Physiology and Chronic Health Evaluation; SOFA: Sequential Organ Failure Assessments

CRP and PCT levels showed no significant correlation between the bacteremic and nonbacteremic groups in terms of medical/ surgical status $(P=0.151$ and $P=0.371)$.

In the nonbacteremic group, PCT levels were not different between patients with intra-abdominal infection and other sources of infection $(P=0.061)$. On the other hand, in bacteremic patients, PCT was significantly higher in intra-abdominal infections $(32.3 \pm 39.9 \mathrm{ng} / \mathrm{dL})$ compared to other infection sources $(7.3 \pm 10.3 \mathrm{ng} / \mathrm{dL})(P=0.013)$. PCT levels in patients with intra-abdominal infection $(14.6 \pm 24.6 \mathrm{ng} / \mathrm{dL})$ were significantly higher compared to other infections $(5.9 \pm 11.4 \mathrm{ng} / \mathrm{dL})$ when all patients were analyzed $(P=0.008)$.

\section{Prognostic value of procalcitonin}

The overall mortality rate was $60.3 \%$. There was no significant difference in mortality between the bacteremic and nonbacteremic groups $(P=0.418)$. We found a statistically significant difference between the bacteremic $(n=41,43.6 \%)$ and nonbacteremic groups $(n=53,56.4 \%)$ in terms of 28 -day mortality rate $(P=0.002)$ [Table 1]. In subgroup analysis, the overall mortality rate for patients with sepsis $(n=29,30.8 \%)$ and severe sepsis/septic shock ( $n=58,61.7 \%$ ) were significantly higher than those diagnosed with SIRS $(n=7,7.4 \%)(P=0.001, P=0.021$, and $P=0.129$, respectively). However, there was no significant difference in 28-day mortality rate among SIRS, sepsis, and severe sepsis/septic shock patients $(P>0.05)$. 


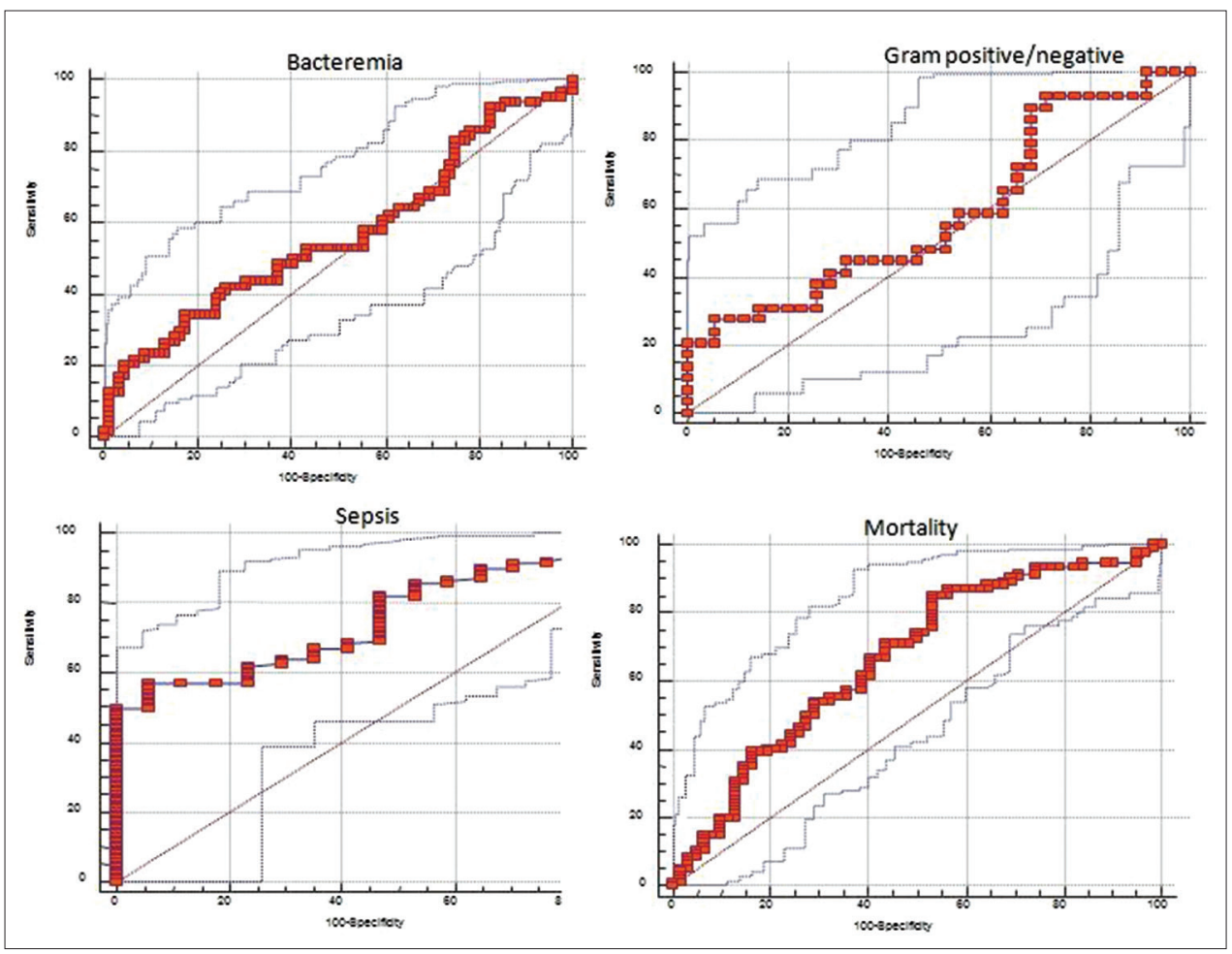

Figure 2: Receiver operating characteristic curves analysis for various cutoff levels of procalcitonin in predicting bacteremia, Gram positive-negative bacteria, sepsis, and mortality. Area under the curve for a null hypothesis was 0.5

Table 2: Diagnostic and prognostic value of procalcitonin

\begin{tabular}{lccccccccc}
\hline Variables & AUC & $\boldsymbol{P}$ & Cutoff* $^{*}$ & Sensitivity (\%)** & Specificity(\%)** & $\begin{array}{c}\text { Positive } \\
\text { LR }\end{array}$ & $\begin{array}{c}\text { Negative } \\
\text { LR }\end{array}$ & $\begin{array}{c}\text { Positive } \\
\text { PV (\%) }\end{array}$ & $\begin{array}{c}\text { Negative } \\
\text { PV(\%) }\end{array}$ \\
\hline Bacteremia & 0.565 & 0.168 & 9.3 & 34.5 & 82.6 & 1.9 & 0.8 & 57.9 \\
Sepsis & 0.764 & $<0.001$ & 2 & 56.8 & 94.1 & 9.7 & 0.5 & 98.7 \\
Mortality & 0.667 & $<0.001$ & 0.8 & 85.1 & 46.8 & 1.6 & 0.3 & 70.8 & 67.4
\end{tabular}

*Youden index was used in determining cut off value, **Presented with 95\% CI. AUC: area under the ROC curve; LR: Likelihood ratio; PV: Predictive value; CI: Confidence interval; ROC: Receiver operating characteristic

Mean PCT levels were significantly higher in nonsurvivors compared to survivors $(10.1 \pm 18.0$ vs. $5.7 \pm 13.7 \mathrm{ng} / \mathrm{dL}$; $P<0.001)$. The significant cutoff value of PCT was determined as $0.8 \mathrm{ng} / \mathrm{ml}$ in predicting mortality. The AUC value for PCT was 0.667 (95\% CI: 0.579-0.756) [Table 2 and Figure 2].

Mean PCT levels, Charlson comorbidity index, APACHE II scores, and SOFA scores of patients with severe sepsis/septic shock were significantly higher than patients with noninfectious SIRS and sepsis. There was a positive but weak correlation between SOFA score and PCT or CRP levels $(P<0.001)$.

\section{Discussion}

PCT has been used extensively in the clinical diagnosis of sepsis before blood cultures. Furthermore, it has been reported that PCT can differentiate bacteremia from inflammatory sepsis in $77 \%$ of cases with other clinical parameters. ${ }^{[1]}$ On the other hand, PCT alone has some limitations in discriminating infectious diseases, and PCT levels may increase in noninfectious conditions. ${ }^{[5]}$ Similarly, our study also demonstrated that PCT levels were not significant in predicting bacteremia. Consequently, we believe that there is a need for further well-designed studies to investigate the diagnostic value of PCT in predicting bacteremia.

In recent years, it has been reported that Gram-positive bacteremia has a higher frequency in the ICU. ${ }^{[12]}$ Suner et al. reported that Gram-positive cocci are the most common pathogen $(42.18 \%)$ in ICU. ${ }^{[13]}$ However, in a multicenter prospective study which was conducted in Greece between 2006 and 2013, it was stated that the most common microorganisms were Gram-negative bacteria in ICU. ${ }^{[14]}$ We also found that the rate of bacteremia for both Gram-negative and Gram-positive bacteria was high, in accordance with the literature. It is expected that the frequency of Gram-positive bacteremia will increase due to the frequent use of antibiotics against Gram-negative bacteria and use of long-term intravascular catheters.

Respiratory tract, urinary tract, and intra-abdominal infections mainly reported sources of sepsis in the ICU. ${ }^{[15,16]}$ In our 
study, the source of infection was similar to the literature as follows: the respiratory tract, the abdomen, the urinary tract, the soft tissue, and the catheter infections, respectively. We believe the incidence of catheter infection was low in our ICU due to effective infection control programs.

It has been suggested that PCT levels may help to distinguish Gram-negative bacterial infections from Gram-positive infections ${ }^{[17]}$ Leli et al. demonstrated that the average level of PCT was $13.8 \mathrm{ng} / \mathrm{mL}$ in Gram-negative bacteremia and $2.1 \mathrm{ng} / \mathrm{mL}$ in Gram-positive bacteremia. ${ }^{[17]}$ However, in our study, PCT levels were higher in Gram-negative bacteremia although the difference was not statistically significant. We believe that more extensive research is needed on this issue.

Some studies suggest that PCT levels may be used for the diagnosis and estimation of severity of sepsis. In a study conducted by Su et al., increased PCT levels were associated with more severe sepsis. ${ }^{[18]}$ Similarly, our study also showed that elevated PCT levels were associated with the diagnosis and severity of sepsis. Therefore, we believe that PCT may be an effective biomarker for diagnosing sepsis and monitoring disease severity.

It has been reported in several studies that serum PCT values are higher in intra-abdominal infections. ${ }^{[19,20]}$ In a meta-analysis, 1827 patients were evaluated, and serum PCT levels were shown to have a significant predictive value in patients with intra-abdominal infections. ${ }^{[19]}$ In our study, significantly higher PCT levels were observed in patients with intra-abdominal infections compared to other sources of infection. Therefore, we believe that PCT should be considered in the assessment of intra-abdominal infections.

Length of stay and mortality were found to be increased in ICU patients with bacteremia. ${ }^{[2]}$ In a study conducted in a tertiary ICU, bacteremia was an independent risk factor for nosocomial infection-related mortality. ${ }^{[22]}$ According to our study results, overall mortality was not significantly different between bacteremic and nonbacteremic patients. On the contrary, 28-day mortality was significantly higher in the nonbacteremic patients. We believe that the low isolation rate of bacteremia in our patients might be as a result of previous antibiotic use. We propose that the short length of hospital stay in our patients with severe sepsis/septic shock in ICU was due to an increase in mortality.

The mortality of sepsis remains high despite all advances in medicine. It is reported that the mortality rate increases with the severity of sepsis. ${ }^{[9]}$ Fialkow et al. showed that the mortality rate of patients was higher in ICU, depending on the severity of illness. ${ }^{[23]}$ There was no significant correlation between the disease severity and 28-day mortality; however, similarly, with the literature, the overall mortality rate was increased in patients with higher severity of illness in our study.

Our study has some limitations. Clinical criteria for sepsis were revised in 2016 by Singer et al. ${ }^{[24]}$ However, our patients were evaluated between 2014 and 2015; therefore, ESICM consensus criteria (2012) were used in the diagnosis of sepsis.

\section{Conclusions}

Although PCT levels did not predict bacteremia in our study, we demonstrated that PCT was an effective biomarker for diagnosing sepsis and in predicting disease severity and mortality. There is a need for further well-designed studies to confirm the diagnostic and prognostic value of PCT in septic patients in critical care.

\section{Financial support and sponsorship}

Nil.

\section{Conflicts of interest}

There are no conflicts of interest.

\section{RefEREnCES}

1. Dellinger RP, Levy MM, Rhodes A, Annane D, Gerlach H, Opal SM, et al. Surviving Sepsis Campaign: International guidelines for management of severe sepsis and septic shock, 2012. Intensive Care Med 2013;39:165-228.

2. Prucha M, Bellingan G, Zazula R. Sepsis biomarkers. Clin Chim Acta 2015;440:97-103.

3. Vazquez-Grande G, Kumar A. Optimizing antimicrobial therapy of sepsis and septic shock: Focus on antibiotic combination therapy. Semin Respir Crit Care Med 2015;36:154-66.

4. Chivate CG, Belwalkar GJ, Limaye RP, Patil RV. Procalcitonin as a marker for the diagnosis of sepsis. Int J Res Med Sci 2016;4:1216-8.

5. Wacker C, Prkno A, Brunkhorst FM, Schlattmann P. Procalcitonin as a diagnostic marker for sepsis: A systematic review and meta-analysis. Lancet Infect Dis 2013;13:426-35.

6. Simon L, Gauvin F, Amre DK, Saint-Louis P, Lacroix J. Serum procalcitonin and C-reactive protein levels as markers of bacterial infection: A systematic review and meta-analysis. Clin Infect Dis 2004;39:206-17.

7. Cho SY, Choi JH. Biomarkers of sepsis. Infect Chemother 2014;46:1-2.

8. Liu D, Su L, Han G, Yan P, Xie L. Prognostic value of procalcitonin in adult patients with sepsis: A Systematic review and meta-analysis. PLoS One 2015;10:e0129450.

9. Li S, Rong H, Guo Q, Chen Y, Zhang G, Yang J, et al. Serum procalcitonin levels distinguish gram-negative bacterial sepsis from gram-positive bacterial and fungal sepsis. J Res Med Sci 2016;21:39.

10. European Committee on Antimicrobial Susceptibility Testing. Version 7.0; 2014.

11. Yap CY, Aw TC. The use of procalcitonin in clinical practice. Proc Singapore Healthc 2014;23:33-7.

12. Anderson DJ, Moehring RW, Sloane R, Schmader KE, Weber DJ, Fowler VG Jr., et al. Bloodstream infections in community hospitals in the $21^{\text {st }}$ century: A multicenter cohort study. PLoS One 2014;9:e91713.

13. Süner A, Karaoğlan I, Mete AO, Namiduru M, Boşnak V, Baydar I, et al. Assessment of bloodstream infections and risk factors in an İntensive Care Unit. Turk J Med Sci 2015;45:1243-50.

14. Koupetori M, Retsas T, Antonakos N, Vlachogiannis G, Perdios I, Nathanail C, et al. Bloodstream infections and sepsis in Greece: Over-time change of epidemiology and impact of de-escalation on final outcome. BMC Infect Dis 2014;14:272.

15. Angus DC, van der Poll T. Severe sepsis and septic shock. N Engl J Med 2013;369:840-51.

16. Lagu T, Rothberg MB, Shieh MS, Pekow PS, Steingrub JS, Lindenauer PK, et al. Hospitalizations, costs, and outcomes of severe sepsis in the United States 2003 to 2007. Crit Care Med 2012;40:754-61.

17. Leli C, Ferranti M, Moretti A, Al Dhahab ZS, Cenci E, Mencacci A, et al. Procalcitonin levels in gram-positive, gram-negative, and fungal bloodstream infections. Dis Markers 2015;2015:701480. 
18. Su L, Feng L, Song Q, Kang H, Zhang X, Liang Z, et al. Diagnostic value of dynamics serum SCD163, sTREM-1, PCT, and CRP in differentiating sepsis, severity assessment, and prognostic prediction. Mediators Inflamm 2013;2013:969875.

19. Qu J, L X, Liu Y, Wang X. Evaluation of procalcitonin, C-reactive protein, interleukin-6 \& serum amyloid A as diagnostic biomarkers of bacterial infection in febrile patients. Indian J Med Res 2015;141:315-21.

20. Watkins RR, Lemonovich TL. Serum procalcitonin in the diagnosis and management of intra-abdominal infections. Expert Rev Anti Infect Ther 2012;10:197-205.

21. Brooks D, Smith A, Young D, Fulton R, Booth MG. Mortality in intensive care: The impact of bacteremia and the utility of systemic inflammatory response syndrome. Am J Infect Control 2016;44:1291-5.

22. Rejeb MB, Sahli J, Chebil D, Khefacha-Aissa S, Jaidane N, Kacem B, et al. Mortality among patients with nosocomial infections in tertiary Intensive Care Units of Sahloul hospital, Sousse, Tunisia. Arch Iran Med 2016;19:179-85.

23. Fialkow L, Farenzena M, Wawrzeniak IC, Brauner JS, Vieira SR, Vigo A, et al. Mechanical ventilation in patients in the Intensive Care Unit of a general university hospital in Southern Brazil: An epidemiological study. Clinics (Sao Paulo) 2016;71:144-51.

24. Singer M, Deutschman CS, Seymour CW, Shankar-Hari M, Annane D, Bauer $\mathrm{M}$, et al. The third international consensus definitions for sepsis and septic shock (Sepsis-3). JAMA 2016;315:801-10. 